\title{
Molecular and metabolic pathways mediating curative treatment of a non- Hodgkin B cell lymphoma by Sindbis viral vectors and anti-4-1BB monoclonal antibody
}

\author{
Minjun Yu, Iris Scherwitzl, Silvana Opp, Aristotelis Tsirigos and Daniel Meruelo*
}

\begin{abstract}
Background: Limitations to current therapies for treating non-Hodgkin B cell lymphoma include relapse, toxicity and high cost. Thus, there remains a need for novel therapies. Oncolytic viral (OV) therapy has become a promising cancer immunotherapy because of its potential effectiveness, specificity and long-lasting immunity. We describe and characterize a novel cancer immunotherapy combining Sindbis virus (SV) vectors and the agonistic monoclonal antibody (mAb) to the T cell costimulatory receptor, 4-1BB (CD137).

Methods: A20 lymphoma was transfected with luciferase and tumor cells were inoculated to BALB/C mice. Tumor growth was monitored by IVIS imaging. Tumor bearing mice were treated with Sindbis virus, a4-1BB Ab or SV plus a4-1BB Ab. On day 7 after treatment, splenocytes were harvested and surface markers, cytokines, and transcription factors were measured by flow cytometry or Elispot. Splenic T cells were isolated and RNA transcriptome analysis was performed. Tumor cured mice were rechallenged with tumor for testing immunological memory.

Results: SV vectors in combination with a4-1BB monoclonal antibody ( $\mathrm{AAb}$ ) completely eradicated a B-cell lymphoma in a preclinical mouse model, a result that could not be achieved with either treatment alone. Tumor elimination involves a synergistic effect of the combination that significantly boosts T cell cytotoxicity, IFNY production, T cell proliferation, migration, and glycolysis. In addition, all mice that survived after treatment developed long lasting antitumor immunity, as shown by the rejection of A20 tumor rechallenge. We identified the molecular pathways, including upregulated cytokines, chemokines and metabolic pathways in T cells that are triggered by the combined therapy and help to achieve a highly effective anti-tumor response.
\end{abstract}

Conclusions: Our study provides a novel, alternative method for B cell lymphoma treatment and describes a rationale to help translate SV vectors plus agonistic $m A b$ into clinical applications.

Keywords: Sindbis virus(SV), a4-1BB, T cell, IFNY, RNA-Seq, IVIS imaging

\footnotetext{
*Correspondence: Daniel.meruelo@nyumc.org

Perlmutter Cancer Center at NYU Langone Health, NYU Gene Therapy

Center, and Department of Pathology, NYU School of Medicine, 550 First

Avenue, New York, NY 10016, USA
}

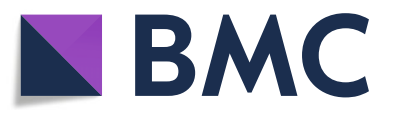

(c) The Author(s). 2019 Open Access This article is distributed under the terms of the Creative Commons Attribution 4.0 International License (http://creativecommons.org/licenses/by/4.0/), which permits unrestricted use, distribution, and reproduction in any medium, provided you give appropriate credit to the original author(s) and the source, provide a link to the Creative Commons license, and indicate if changes were made. The Creative Commons Public Domain Dedication waiver (http://creativecommons.org/publicdomain/zero/1.0/) applies to the data made available in this article, unless otherwise stated. 


\section{Background}

Chemotherapy and immunotherapy (monoclonal antibodies (mAbs) and CAR-T therapy) have been used to treat non-Hodgkin B cell lymphoma. For both conventional chemotherapy and immunotherapy, tumor relapse is a common problem [1]. Establishment of a potent, safe, but also long-lasting immune response is a major goal of $\mathrm{B}$ cell lymphoma treatment. CAR-T therapy is a novel immune therapy used to treat diffuse large B cell lymphoma. However, major drawbacks to current CAR-T therapy include: risk of immune incompatibility for allogeneic CAR-T [2]; quality control for harvesting self $\mathrm{T}$ cells; time consumption for processing to autologous CAR-T [3]; off-target effects; the possibility that the treatments causes a cytokine storm and high cost. Therefore, there is a need to investigate alternative and reliable methods for treating B cell lymphoma.

Oncolytic virus (OV) therapy has become a novel immunotherapeutic approach to treat cancer. A rationale for oncolytic virus is that they can infect and lyse the tumor cells [4]. They have been made to selectively replicate in tumor cells either through the direction of tumor specific promoters or through direct intratumoral administration. Most OVs encounter a number of barriers to systemic administration. Once lysed by OVs, tumor cells release tumor associated antigens (TAAs) [5] that can stimulate cytotoxic T cells. OV infection also induces an inflammatory response that helps to trigger an immune anti-tumor response [6]. Several OV clinical trials are underway and have shown promising results [7]. However, whether OV therapy can effectively treat tumors that they are unable to infect remains an unresolved limitation.

Sindbis virus (SV) belongs to alphavirus genus and is one type of OV $[4,8]$. Though it does not lyse infected tumor cells, it can cause their apoptotic death. It offers several important benefits. SV is known as one of the least virulent alphaviruses with clinical signs and symptoms usually unapparent [9]. It has been estimated that there are 17 times more subclinical than symptomatic SV infections [10]. In general, when symptoms do occur in humans they consist of a self-limiting, mild, febrile disease with vesicular exanthema and arthralgia from which most patients recover within 14 days [11]. The disease is in part self-limiting because $\mathrm{SV}$ is an RNA virus that does not integrate in the host genome and hence its presence is transitory [12]. The lack of an integrative step in its replication cycle also avoids insertional mutagenesis risks. In addition, our SV vectors were generated from the laboratory strain AR339, which is not known to cause disease in humans [13]. We further attenuated these vectors by rendering them replicationdefective [14].
SV vectors can target tumors systemically and can reach metastatic tumor cells throughout the body. They can target tumors without infecting normal tissues [8]. However, susceptibility to infection by SV vectors depends on a number of factors including laminin receptor expression [15] and distribution, as well as, defects in IFN signaling in tumors [16]. Here we document that $\mathrm{SV}$ vectors can effectively help cure tumors that they are unable to infect.

Our present studies use an antibody directed at 4-1BB (CD137, TNFRSF9), a T cell costimulatory molecule. 41BB agonist stimulation greatly enhances NK and cytotoxic $\mathrm{T}$ cell activity. There are preclinical studies showing that $\alpha 4-1 B B$ effectively treats lymphoma and that depletion of Treg cells enhances the therapeutic effect of $\alpha 4$ 1BB [17]. The A20 tumor cells we use in the present study were derived from a spontaneously arising reticulum cell sarcoma (a non-Hodgkin lymphoma) in a BALB/c mouse.

Previously, we used SV carrying NYESO-1, which encodes the cancer testis TAA, NYESO-1, to cure CT26 tumors expressing NYESO-1 [18]. Here we show that systemically disseminated A20 lymphoma can be completely cured by SV plus $\alpha 4-1 B B$ mAb combination therapy without the need to produce a SV that encodes a TAA known to be present in the A20 lymphoma cells. Further, neither intratumoral injection of the SV vectors nor infection of the tumors is required as the A20 B lymphoma cells used in the current model are resistant to SV infection.

One difference in the current study, compared with those we previously published, is the use of SV vector combination therapy that involves an agonistic mAb for a costimulatory receptor versus targeting checkpoint blockade molecules such as CTLA4 and PD-1. Here we show that agonistic mAbs in combination with SV vectors trigger a cascade of events that results in curative results.

Our findings reveal the potential of SV combination therapy to cure tumors for which TAAs are completely unknown.

\section{Methods}

Firefly luciferase (Fluc)-expressing A20 cells generation A20 cells were transfected with pGL4-neo_Fluc plasmid (Promega) by electroporation via Nucleofector ${ }^{\text {TM }}$ kit $\mathrm{V}$ (Lonza). Fluc-A20 cell clones were selected and maintained in RPMI1640 (Cellgro) + 10\% FBS (Gibco) + $250 \mu \mathrm{g} / \mathrm{ml} \mathrm{G} 418$ (Gibco). One A20 clone stably expressed fLuc and was used for tumor inoculation and consecutive experiments.

\section{SV production}

SV-LacZ production and titering were done the same as previously described [18]. 


\section{SV-GFP infection}

A20 cells and control BHK cells were infected by SV carrying GFP for $1 \mathrm{~h}$. The GFP expression was observed the next day by fluorescence microscopy.

\section{A20 tumor inoculation and In Vivo Imaging System (IVIS) imaging}

$3 \times 10^{6}$ fLuc-A20 cells were inoculated to BALB/C mice by i.p injection. Tumor growth was monitored as previously described [18].

\section{SV and a4-1BB Ab treatment}

Treatment was started after successful tumor inoculation (4 days after tumor cell injection, confirmed by IVIS imaging). Tumor growth was measured every week by noninvasive bioluminescent imaging. SVLacZ was injected 4 times per week, for totally 3 weeks. The virus $\left(10^{7}-10^{8} \mathrm{TU} / \mathrm{mL}\right)$ in a total volume of $500 \mu \mathrm{L}$ was i.p. injected. For 2 groups $(41 \mathrm{BB}$ and $\mathrm{SV}$ plus $41 \mathrm{BB}), 350 \mu \mathrm{g} /$ mouse $41 \mathrm{BB} \mathrm{Ab}$ was injected 3 times/week for 2 weeks. InVivoMAb anti-mouse 4$1 \mathrm{BB}$ was ordered from BioXCell (Clone: LOB12.3, Cat.No. BE0169). In low dose treatment protocol, SVLacZ was injected i.p. 3 times per week, for totally 3 weeks. 41BB Ab $(50 \mu \mathrm{g} /$ mouse $)$ was injected once a week for 3 weeks.

\section{Elispot}

Mouse IFN $\gamma$ ELISPOT was performed according to the manufacturer's protocol (BD Biosciences). $2 \times 10^{5}$ splenocytes or $1 \times 10^{5} \mathrm{~T}$ cells were plated per well $\mathrm{O} / \mathrm{N}$ in RPMI supplemented with 10\% FBS. For a positive control, splenocytes were stimulated with $5 \mathrm{ng} / \mathrm{ml} \mathrm{PMA}+$ $1 \mu \mathrm{g} / \mathrm{ml}$ Ionomycin.

\section{Flow cytometry}

Fluorochrome-conjugated antibodies against mouse CD3, CD4, CD8, CD25, CD44, CD62L, ICOS, CD11a, ICAM-1 were purchased from Biolegend (San Diego, CA). Fluorochrome-conjugated antibodies against mouse Foxp3, EOMES and CCR5 were purchased from Thermofisher. BUV395 conjugated antibody against mouse CD8a was purchased from BD Biosciences. For surface staining, cells were washed and stained with anti-mouse direct conjugated antibodies. Cells were analyzed using the LSRII flow cytometer (BD Biosciences) and data were analyzed using Flowjo software (Treestar, Ashland, OR). For intracellular cytokines staining, stimulated cells were fixed with cytofix/cytoperm solution (BD Biosciences), permeablized with perm/wash buffer (BD Biosciences) and stained with anti-mouse IFN $\gamma$ antibodies. For nuclear antigen, cells were fixed and permeabilized by Foxp3 fixation/permeabilization buffer (eBioscience) and stained with anti-Foxp3, T-bet, Ki67 and EOMES antibody.

\section{RNA isolation and transcriptome analysis}

Total RNA was harvested by RNAeasy isolation kit (Qiagen, Valencia, CA). For each group, $3 \mathrm{BALB} / \mathrm{C}$ mice were used as biological repeats. RNA-seq was performed by NYUMC Genome Technology Center (GTC). To identify significant differences in expression between any pair of groups, differential expression analysis was performed using Deseq2 and an adjusted $p$ value cutoff of 0.05 was applied [19] $(\mathrm{q}<0.05)$. To increase stringency, only genes with a $\log 2$ fold change $\geq 1$ (upregulated) or $\leq$ -1 (downregulated) were selected for further analysis. Gene cluster analysis was performed by DAVID analysis using the selected differentially expressed genes [20,21]. RNA-seq results (normalized counts) were used as input to perform with Gene Set Enrichment Analysis (GSEA) [22]. Molecular Signatures Database (MSigDB)v4.0 were used as screening database. For each gene, the gene expression value is normalized by the relative $\log 2$ fold change compared to the median value of this gene. Expression heatmap is drawn by Morpheus (https://software.broadinstitute.org/morpheus/). Cannonical pathway and disease and biological functional analysis were generated by ingenuity pathway analysis (IPA; Ingenuity Systems, Redwood City, CA) using the statistical differential expressed genes list. To increase the sample representativeness, for IPA, we choose nominal $p<0.05$ as cutoff value.

\section{Tumor infirtrating lymphocyte (TIL) harvest}

To investigate the phenotype of TIL, all treatments were started 11 days after tumor inoculation, After 7 days treatment, tumor mass was harvested and the phenotype of TIL were analyzed as previously described [18].

\section{T cell seahorse assay}

$\mathrm{T}$ cells were isolated from spleen by using pan $\mathrm{T}$ cell isolation kit (Stemcells). T cells were plated at $6 \times 10^{5}$ cells/well in 24 well plate. Oxygen consumption rate (OCR) and excellular acidification rate (ECAR) were measured by Agilent Seahorse XFe24.

\section{Statistical analysis}

For the two group comparison, statistical difference was determined by unpaired two tail Student $\mathrm{t}$-test. The multiple sample comparison was analyzed by one way ANOVA. $P<0.05$ was determined to be significant for all experiments. All values were calculated with Excel (Microsoft) and Prism software (GraphPad). 


\section{Results}

SV and a4-1BB mAb combination completely cured A20 lymphoma

To explore if SV has therapeutic effect on tumors not targeted or infected by SV vectors, we used the A20 B cell lymphoma, which is highly resistant to SV infection (Additional file 1: Figure S1).

To monitor tumor growth in vivo, a firefly luciferase (f-Luc) expression vector was transfected into the A20 lymphoma cell line by electroporation. A stable f-Luc expressing A20 clone was isolated through G418 selection. We inoculated $3 \times 10^{6} /$ mouse f-Luc A20 tumor cells by intraperitoneal (i.p.) injection. Tumor growth was monitored by IVIS imaging once per week. Tumors were successfully established after 4 days inoculation (Fig. 1a). After tumors were established, SV and $\alpha 4-1 \mathrm{BB}$ $\mathrm{mAb}$ treatment started (designated as day 0). We used a therapeutic protocol similar to that previously described [18]. SV plus $\alpha 4-1 B B$ mAb combination achieved the best therapeutic effect (Fig. 1b). All mice in that group showed complete tumor regression in 2 weeks. Although both SV or $\alpha 4-1 B B$ treatments alone achieved obvious therapeutic effects compared with untreated mice, they were not as effective as the combination and a fraction of mice in these two groups eventually succumbed to tumor (Fig. 1c).

\section{SV alone and SV plus a4-1BB mAb stimulated cell cycle progression, cytokine production, and activation}

In our study, SV significantly inhibited tumor growth by day 7 (Fig. 1a). T cells play a critical role in SV induced anti-tumor immunity. $\mathrm{T}$ cell response reaches a peaked on day 7 after infection [18]. To explore how SV induced $\mathrm{T}$ cell responses that help to eradicate A20 lymphoma, RNA-Seq was performed using purified splenic $\mathrm{T}$ cells from all groups on day 7. Compared with untreated samples, we identified 271 genes upregulated $(\mathrm{q}<0.05$ and Log2 Fold Change $\geq 1$ ) and 28 genes downregulated $(q<0.05$ and Log2 Fold Change $\leq-1)$ in the SV infected group through Deseq2 analysis (Fig. 2a, Additional file 2: Table S1). NIH DAVID cluster analysis was performed using the upregulated gene list. Enriched clusters were ranked based on enrichment score. Cell cycle gene cluster achieved the highest enrichment score (Fig. 2b, Additional file 1: Figure S2A). This result was confirmed by KEGG gene set enrichment analysis (GSEA) (Additional file 1: Figure S2B). Cell cycle gene set ranks as the highest (enrichment score $=0.64$, FDR q value $=0.1$, nominal $p$ value $=0)$. These results indicate that SV infection enhances $\mathrm{T}$ cell cell cycle progression. SV induced upregulation of a series of cytokine and chemokine/chemokine receptors (Fig. 2c, left). To identify cytokines/chemokines that are upregulated by the administration of SV vectors, we compared SV plus $\alpha 4-1 \mathrm{BB}$ mAb versus $\alpha 4$ 1BB mAb (Fig. 2c, right). CCL8, IL-4, IL-13 and IL-21 were among those RNAs whose expression was upregulated by SV treatment. IL-21 anti-tumor effect is dependent on the activation of T, B and NK cells [23]. IL-4, IL-10, IL-21 upregulation is consistent with previous reports $[24,25]$.

In addition, Ingenuity Pathway Analysis (IPA) indicates that SV treatment enhances T cell movement by altering the expression of a number of molecules involved migration (Additional file 3: Table S2, Additional file 1: Figure S2C), including a number of chemokines and chemokine receptors.

To understand why SV plus $\alpha 4-1 \mathrm{BB}$ mAb achieves the best therapeutic effect, we ran Deseq2 analysis for SV plus $\alpha 4-1 \mathrm{BB}$ mAb vs. untreated samples. We identified 1046 upregulated genes $(\mathrm{q}<0.05$ and Log2 Fold Change $\geq 1)$ and 877 downregulated genes $(q<0.05$ and Log2 Fold Change $\leq-1$ ) in the SV plus $\alpha 4-1 B B$ mAb group (Fig. 2a, Additional file 4: Table S3). We also compared $\mathrm{T}$ cells from animals treated with $\mathrm{SV}+$ $\alpha 4-1 \mathrm{BB}$ mAb vs. treated with SV only and found 316 upregulated genes $(p<0.05$ and Log2 Fold Change $\geq 1)$ and 439 downregulated genes $(p<0.05$ and Log2 Fold Change $\leq-1)$ in the $\mathrm{SV}+\alpha 4-1 \mathrm{BB} \mathrm{mAb}$ treated group (Fig. 2a, Additional file 5: Table S4). Next, we ran NIH DAVID analysis using the upregulated gene list. In both comparisions, cell cycle genes upregulation is the highest enrichment cluster [although $\mathrm{SV}+\alpha 4-1 \mathrm{BB}$ $\mathrm{mAb}$ vs. SV has a lower enrichment score compared with SV plus $\alpha 4-1 B B$ mAb vs. untreated samples (Fig. $2 \mathrm{~b}$ and Additional file 1: Figure S3). This indicates that $\mathrm{SV}+\alpha 4-1 \mathrm{BB} \mathrm{mAb}$ induced more potent $\mathrm{T}$ cell cycle progression compared with SV only. T cell proliferation is critical for an effective anti-tumor response against A20 lymphoma. The CD4/CD8 T cell ratio in untreated mice decreased markedly by day 28 after tumor inoculation (Additional file 1: Figure S4AB). In addition, Treg/CD8 $\mathrm{T}$ cell ratio increased by day 28, indicating impairment of $\mathrm{T}$ cell function (Additional file 1: Figure S4C-D). In other groups the $\mathrm{T}$ cell ratio remained constant due to proliferation.

CD69 is the earliest marker of immune system activation. SV plus $\alpha 4-1 \mathrm{BB}$ mAb treatment synergistically upregulated CD69 on day 2 (Fig. 2d). Additionally, KEGG GSEA indicates that $\mathrm{T}$ cell receptor signaling gene sets were enriched when comparing $S V+\alpha 4-1 B B$ vs untreated samples (enrichment score $=0.35$, Normalized Enrichment Score $(\mathrm{NES})=1.56, \quad \mathrm{FDR}$ q value $=0.17$, nominal $p$ value $=0$ ) (Fig. 2e).

SV plus a4-1BB mAb stimulated cytotoxic $T$ cell function To investigate the antitumor cytotoxicity of SV/ 44 1BB treated splenocytes, we co-cultured f-Luc A20 


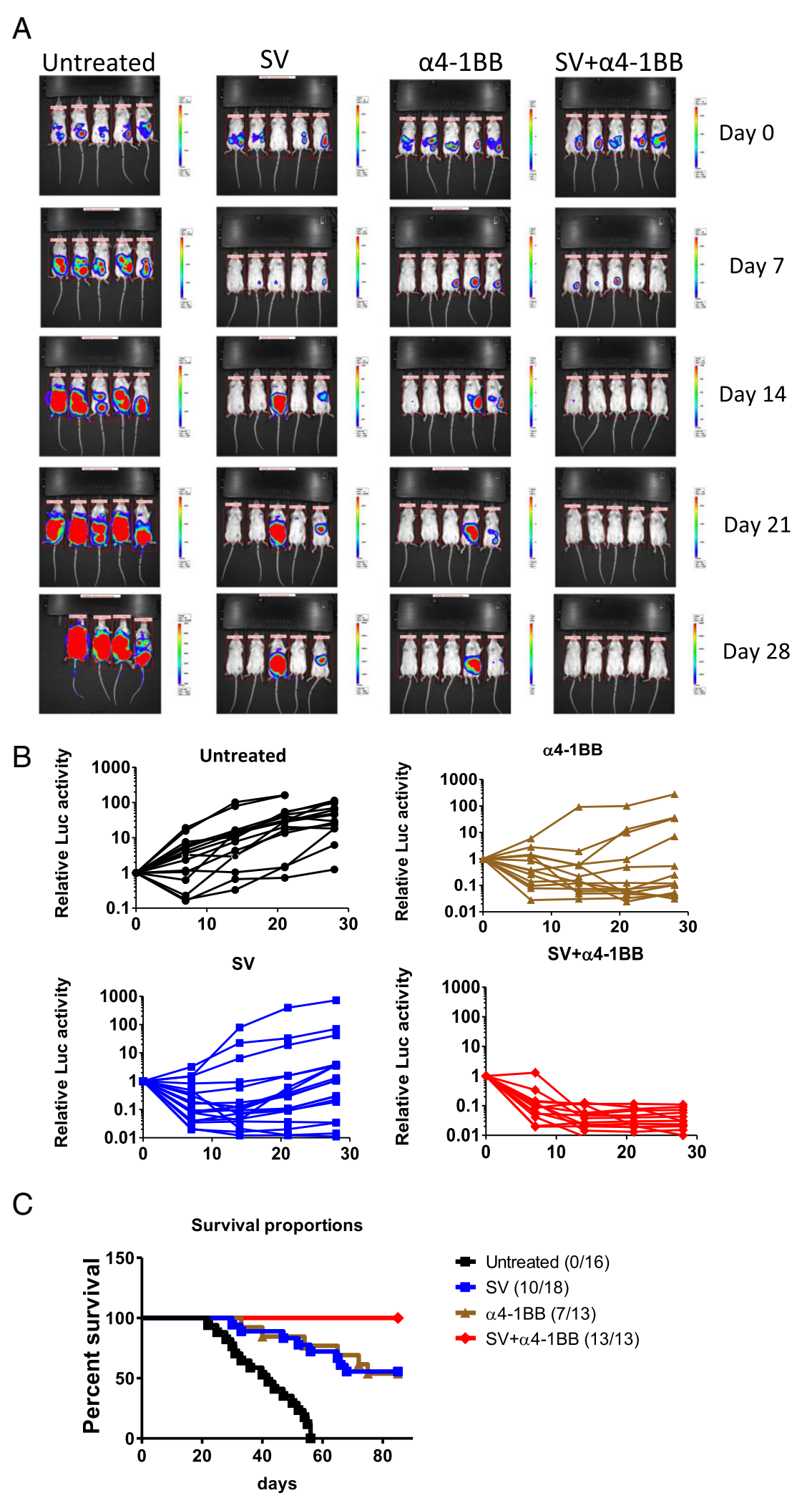

Fig. 1 (See legend on next page.) 
(See figure on previous page.)

Fig. 1 Sindbis virus (SV) and a4-1BB combination completely cured BALB/C mice A20 lymphoma. a Representative bioluminescence images of groups as indicated. Intensity scale, day 0, 7, 21, min: 400, max:7000; day 14, min: 100, max: 1000; day 28, min: 3000, max: 50000. b Tumor growth was measured by relative firefly luciferase (fLuc) activity (normalized to day 0 fLuc activity). Untreated, $n=16 ; \mathrm{SV}, n=18 ; \mathrm{a} 4-1 \mathrm{BB}$ Ab, $n=13$; SV plus a4-1BB Ab, $n=13$. c Survival curve of all groups (the ratio is shown as survived number/total number)

lymphoma cells with splenocytes on day 7. The ratios explored between splenocytes and tumor cell were 40: 1, 20:1, 10:1. SV plus $\alpha 4-1 B B$ treated splenocytes demonstrated the highest cytotoxicity among all groups, as calculated by the reduction of f-Luc activity (Fig. 3a). To understand if this response is induced by TAA or anti-viral immunity, the same experiment was performed using mice under treatment but without tumor inoculation. We found that SV plus $\alpha 4$ $1 \mathrm{BB}$ achieves the same effect as the combination treatment with tumor inoculation. This indicates that anti-tumor response on day 7 was not tumor specific. Accordingly, NKG2D, granzyme $B$ and perforin were highly expressed in CD8 $\mathrm{T}$ cells from $\alpha 4-1 \mathrm{BB}$ treated mice. In addition, SV plus $\alpha 4-1 B B$ in combination induced the highest expression of NKG2D and granzyme $\mathrm{B}$ in CD8 $\mathrm{T}$ cells. NKG2D, granzyme $\mathrm{B}$ and perforin upregulation was tumor independent because the same pattern was observed in all treatments without tumor inoculation (Fig. 3b, c). Correspondingly, IPA indicates that gene sets of cytotoxic $\mathrm{T}$ cell development are significantly upregulated in SV plus $\alpha 4$ 1BB mAb. These genes include Gzmb (granzyme B), Prf1 (perforin) and Klrk1 (NKG2D) (Fig. 3d). These data indicate that SV plus $\alpha 4-1 \mathrm{BB}$ mAb markedly enhanced cytotoxic $\mathrm{T}$ cell activity.

\section{SV plus a4-1BB mAb induced IFNy production from T cells}

Other upregulated genes in the SV plus $\alpha 4-1 \mathrm{BB} \mathrm{mAb}$ combined treatment include STAT4 (Fig. 3d) and IL12rb1 (Fig. 4d), which are required for the development of Th1 cells from naive CD4+ T cells and IFNy production (Fig. 3d) in response to IL-12 [26]. Consistent with this observation, splenocytes from SV plus $\alpha 4-1 \mathrm{BB} \mathrm{mAb}$ treatment produced significantly higher number of IFN $\gamma$ spots compared with other groups, reaching peak production on day 7 (Fig. 4a, upper panel). After day 7, the response dampened but still remained at the highest level compared with other groups (Fig. 4a, lower panel). This is in line with increased IFNy RNA levels. To identify if TAA or viral antigen induces IFN $\gamma$ production on day 7 , the same experiment was performed in mice not inoculated with tumor cells. For both SV or SV plus $\alpha 4-1 \mathrm{BB}$ treatment, the presence or absence of tumor did not significantly affect IFN $\gamma$ levels (Additional file
1: Figure S5), confirmeing that IFNY production on day 7 was mainly an anti-viral response. To identify whether $\mathrm{T}$ cells or antigen presentation cells (APCs) play the major role in IFN $\gamma$ production, we harvested $\mathrm{SV}$ treated splenic $\mathrm{T}$ cells and naive $\mathrm{T}$ cells respectively. $\mathrm{T}$ cells from SV treated mice were co-cultured with naive APCs. Conversely, APCs from SV treated mice were cultured with naive $\mathrm{T}$ cells. $\mathrm{T}$ cells from $\mathrm{SV}$ treated mice produced IFNY when co-cultured with naive APC. Naive $\mathrm{T}$ cells produce much less IFNY spots when cultured with SV infected APC. However, neither $\mathrm{T}$ cell nor APC alone could produce elevated numbers of IFN $\gamma$ spots. These observations indicate that $\mathrm{T}$ cells play the dominant role in $\mathrm{IFN} \gamma$ production during SV infection (Additional file 1: Figure S6A). APCs are essential for helping $\mathrm{T}$ cells to produce IFN $\gamma$.

Next, to identify whether CD4 or CD8 T cells produce IFN $\gamma$, flow cytometric analysis was performed for cytokine analysis. Among splenocytes, 2-2.5\% SV plus $\alpha 4-$ $1 \mathrm{BB}$ mAb treated CD4 T cells produced IFN $\gamma$, which is significantly higher than other groups. Very low percentages of CD8 $\mathrm{T}$ cells produced IFN $\gamma$ in all groups (Fig. $4 \mathrm{~b})$. There were much less IFN $\gamma$ producing $\mathrm{T}$ cells after removing APC (Fig. 4b). Also, there was no difference among all groups for IFN $\gamma$ production. This suggests that $\mathrm{T}$ cell-APC interaction is essential for IFN $\gamma$ production.

To test the antitumor IFN $\gamma$ production activity of the purified $\mathrm{T}$ cells, they were co-cultured for $5 \mathrm{~h}$ with A20 cells, which express major histocompatibility complex (MHC) I and II molecules [27]. Only CD4 T cells from the SV plus $\alpha 4-1 B B$ mAb group produced IFN $\gamma$ after co-culture (Fig. 4c, Additional file 1: Figure $\mathrm{S} 6 \mathrm{~B})$. This indicates that SV plus $\alpha 4-1 \mathrm{BB} \mathrm{mAb}$ induces anti-tumor IFN $\gamma$ production activity. Besides IFN $\gamma$, several Th1 associated genes were also upregulated in the $\mathrm{T}$ cells from SV plus $\alpha 4-1 \mathrm{BB}$ mAb treated groups. These include Ccr5, Cxcr3, Havcr2(Tim3), IL12rb1 and Klrc1 (Fig. 4d).

T-bet is the key transcription factor which is essential for type 1 immune response (IFN $\gamma$ production, $\mathrm{T}$ cell cytotoxicity) and memory $\mathrm{T}$ cell differentiation. In correspondence with the IFN $\gamma$ expression findings, we observed that SV plus $\alpha 4-1 B B$ mAb coordinately upregulates T-bet in T cells on day 7 (Fig. 4e). This suggests that SV helps $\alpha 4-1 B B$ boost the type 1 immune 
A

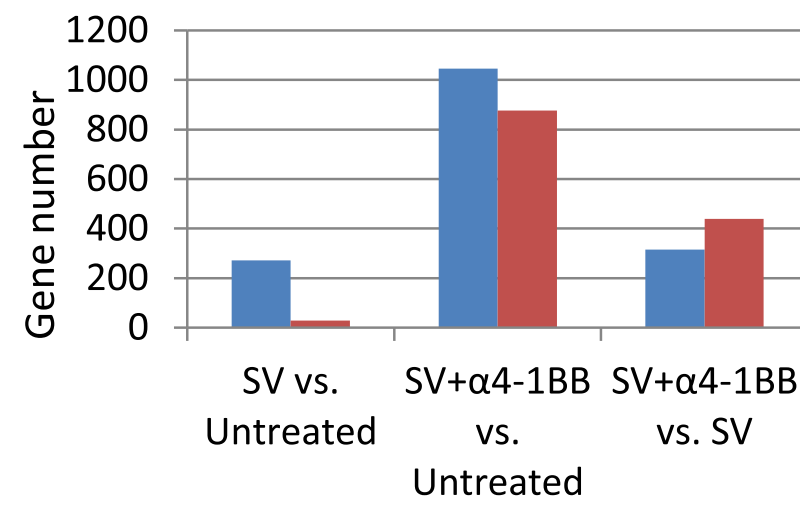

B

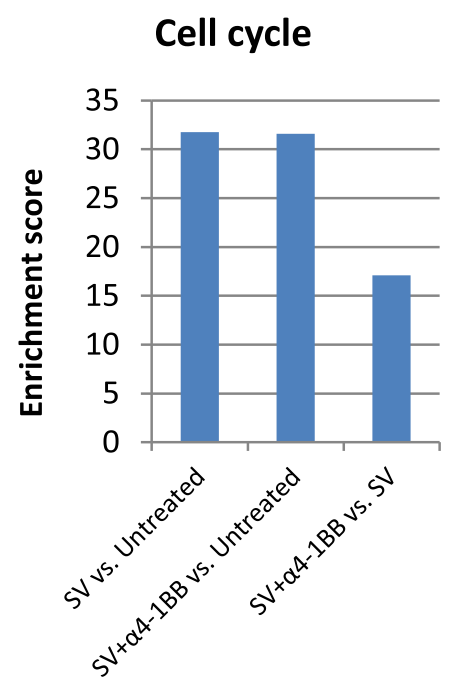

D

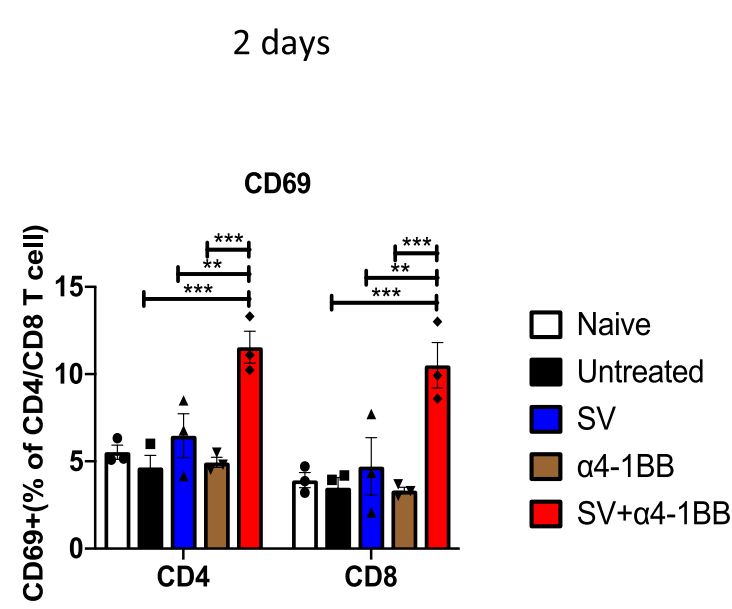

\section{Upregulated}

Downregulated
C

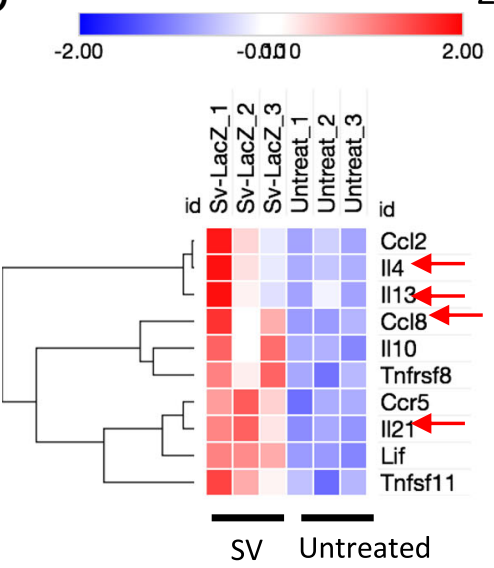
Z score
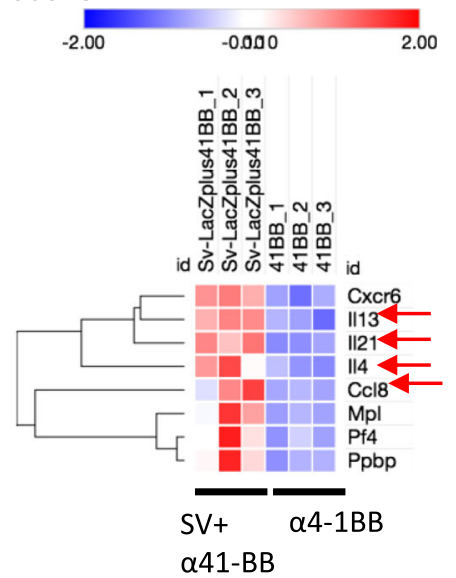

E

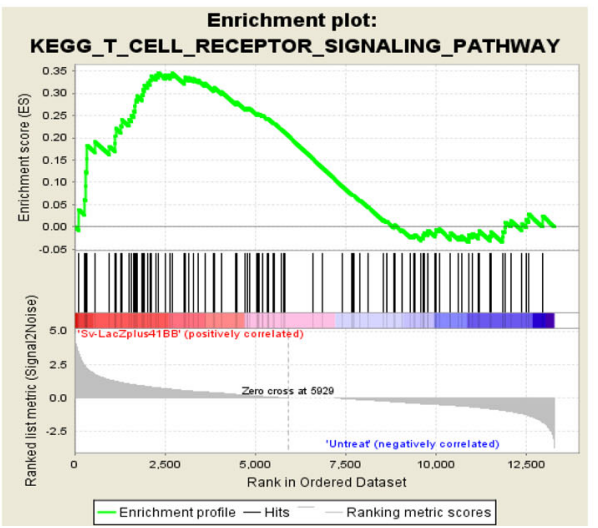

Enrichment score (ES): 0.35

Normalized Enrichment Score (NES) $=1.56$

Nominal $p$ value: 0

False discovery rate (FDR) q-value:0.17

Fig. 2 (See legend on next page.) 
(See figure on previous page.)

Fig. 2 SV alone and SV plus a4-1BB mAb stimulated cell cycle progression, cytokine production, and activation. a The numbers of significant differential (SD) expressed genes (upregulated and downregulated) of SV vs. untreated are as indicated. SD expressed genes were selected based on Deseq2 analysis $(q<0.05), \quad\left|\log _{2} F C\right| \geq 1$. b The enrichment scores for gene cluster of cell cycle for SV vs. untreated, SV+ a4-1BB vs. untreated and SV+ a4-1BB Vs. SV respectively ("cell cycle" is the gene cluster with the highest enrichment score for these 3 comparisons). c The heat map representing SD expressed cytokine and chemokine genes (left, SV vs. untreated; right, SV+ a4$1 \mathrm{BB}$ vs.a4-1BB, Log2FC $\geq 1$ ). Expression values are shown by Z-score. Genes are hierarchically clustered by one minus Pearson correlation. Red arrow, Ccl8, IL4, IL13 and IL21 expression. d The percentage of CD69 ${ }^{+} \mathrm{T}$ cells from all groups on day 2 after starting treatment was measured by flow cytometry. e GSEA enrichment plot of KEGG (SV + a4-1BB vs. untreated) TCR receptor signaling pathway. * $p<0.05$; **, $p<0.01,{ }^{* * *}, p<0.001$

response, which is critical for controlling tumor growth. $\mathrm{SV}$ or $\alpha 4-1 \mathrm{BB} \mathrm{mAb}$ alone could not induce high IFNy production due to low T-bet upregulation. Eomesodermin (EOMES), another important transcription factor, is upregulated in activated $\mathrm{T}$ cells and is essential for memory CD8 $\mathrm{T}$ cell development. Both $\alpha 4-1 \mathrm{BB} \mathrm{mAb}$ and SV plus $\alpha 4-1 \mathrm{BB} \mathrm{mAb}$ induced high expression of EOMES on day 7 (Fig. 4f). The lack of both T-bet and EOMES results in a lower expression of CXCR3 in T cells and a drastic decrease in the number of tumor-infiltrating $\mathrm{T}$ cells [28]. Our data are consistent with these observations. We find elevated CXCR3 (Fig. 4d), T-bet and EOMES (Fig. 4e and $\mathrm{f}$ ) in T cells of the combined SV plus $\alpha 4-1 \mathrm{BB}$ mAb treated animals.

\section{SV and a4-1BB mAb stimulated chemotaxis, adhesion and} enhanced $T$ cell infiltration and activation in tumor

Through RNA-Seq, a series of chemokines and chemokine receptors have been identified to be upregulated in SV plus $\alpha 4-1 \mathrm{BB}$ mAb (Fig. 5a). Among those molecules, CCR5 upregulation was confirmed by flow cytometry (Fig. 5b). CCR5 potentiates CD4 T helper cell functions boosting overall anti-tumor responses [29]. We found SV plus $\alpha 4-1 B B$ significantly upregulates CD11a and ICAM-1(CD54). These two adhesion molecules are highly expressed on activated $\mathrm{T}$ cells. LFA-1 (CD11a/CD18)-ICAM-1 interaction is essential for the formation of immune synapses between $\mathrm{T}$ cell and APC [30]. LFA-1 and ICAM-1 are also required for $\mathrm{T}$ cell- $\mathrm{T}$ cell homotypic aggregation and activation $[31,32] . \alpha 4-1 \mathrm{BB} \mathrm{mAb}$ stimulation induced significant upregulation of CD11a and ICAM-1 in both CD4 and CD8 $\mathrm{T}$ cells whereas SV does not (Fig. 5c-e). In addition, $\mathrm{T}$ cell costimulatory molecule, OX40, was also significantly upregulated in $\mathrm{T}$ cells of mice treated with $\alpha 4-1 B B$. (Fig. 5f, left). OX40 engagement promotes effector $\mathrm{T}$ cell function and survival [33]. ICOS, another CD4 $\mathrm{T}$ cell costimulatory molecule, was upregulated in SV or $\alpha 4-1 B B$ alone but upregulated most in the SV plus $\alpha 4-1 \mathrm{BB}$ combination treatment, suggesting a synergistic effect exists (Fig. 5f, right).
TIL play a critical anti-tumor role and is an important marker for prognosis. Compared with untreated, the percentage of CD3 and CD8 T cells were increased about 2 fold after combination treatment (Fig. $5 \mathrm{~g}$ ). Ki67 were upregulated in those $\mathrm{T}$ cells which indicated active division (Additional file 1: Figure S7A). For untreated TIL, the frequency of Foxp3+ Treg cells was the highest (Additional file 1: Figure S7B) and CD8/Treg ratio was the lowest (Fig. 5h). Treatment enhanced the T-bet and EOMES expression in T cells (Additional file 1: Figure S7C,D). NKG2D and granzyme B were highly upregulated in tumor infirtrating CD8 T cells (Fig. 5i, Additional file 1: Figure S7E). Overall, these data indicate that combination treatment enhanced $\mathrm{T}$ cell infiltration, division, activation, cytotoxicity and downregulated the inhibitory Treg population.

\section{SV and a4-1BB mAb synergistically enhanced oxidative phosphorylation}

$\mathrm{T}$ cell activation requires a quick consumption of energy through both enhanced glycolysis and oxidative phosphorylation [34]. Metabolic switch is a major feature of $\mathrm{T}$ cell activation and memory $\mathrm{T}$ cell development [35]. GSEA KEGG analysis identified that the glycolysis gene set is upregulated in SV plus $\alpha 4-1 \mathrm{BB}$ vs. untreated samples (Fig. 6a). This process quickly produces ATP and supports $\mathrm{T}$ cell migration and cytotoxicity in hypoxic or acidific microenvironments. IPA confirms that SV plus $\alpha 4-1 B B$ mAb synergistically enhanced oxidative phosphorylation (Fig. 6b).

We assessed both oxygen consumption rate (OCR, represents oxidative phosphorylation) and extracellular acidification rate (ECAR, represents glycolysis) of all groups (Fig. 6c). Compared with other groups, SV plus $\alpha 4-1 B B$ significantly increased both OCR and ECAR. This indicates that both glycolysis and oxidative phosphorylation are activated in $\mathrm{T}$ cells of animals treated with SV plus $\alpha 4-1 B B$.

\section{SV plus low dose a4-1BB mAb cured A20 tumor bearing mice}

To reduce the potential risk of cytotoxicity and expense of treatment with SV vectors plus $\alpha 4-1 B B$, we 


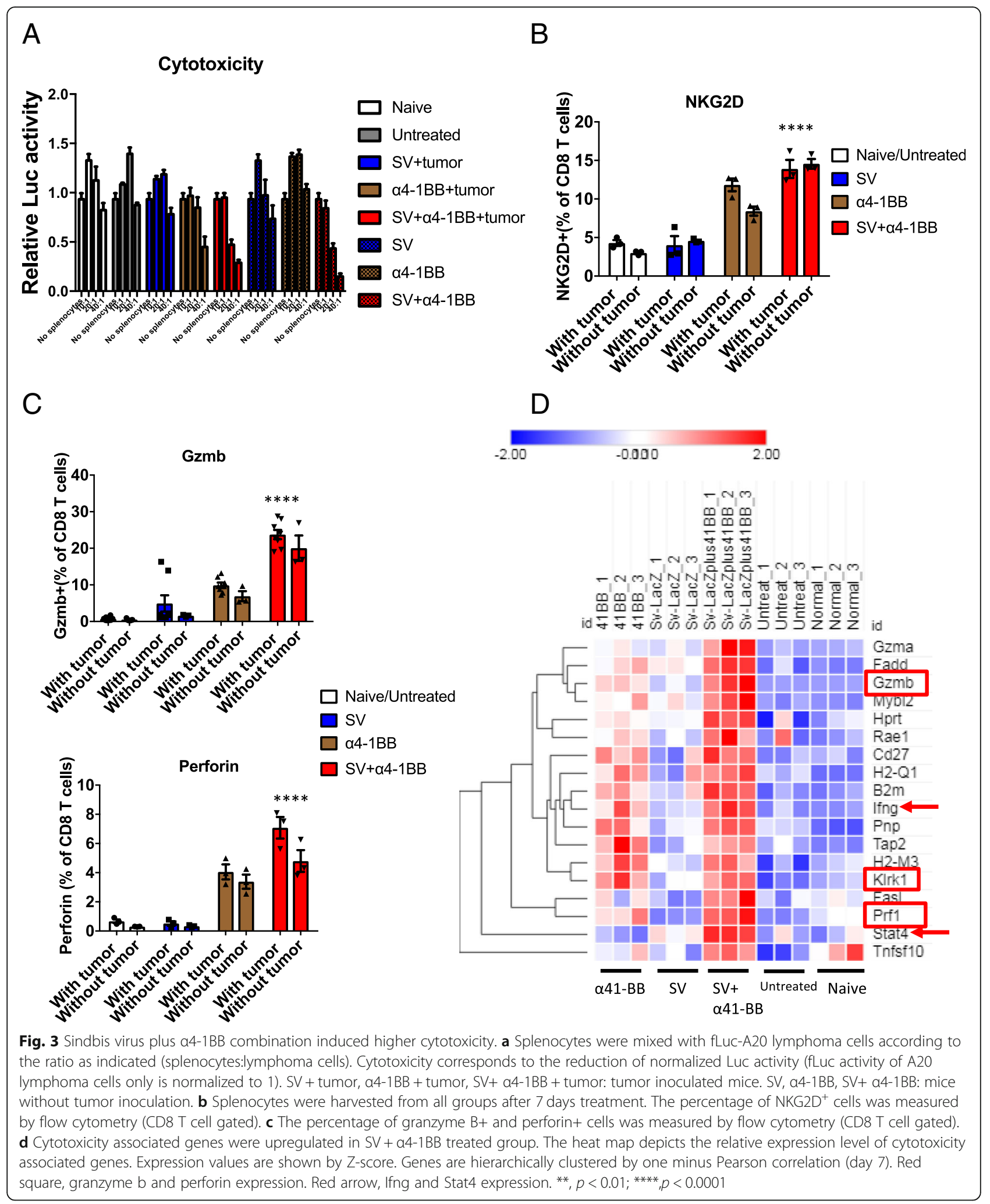




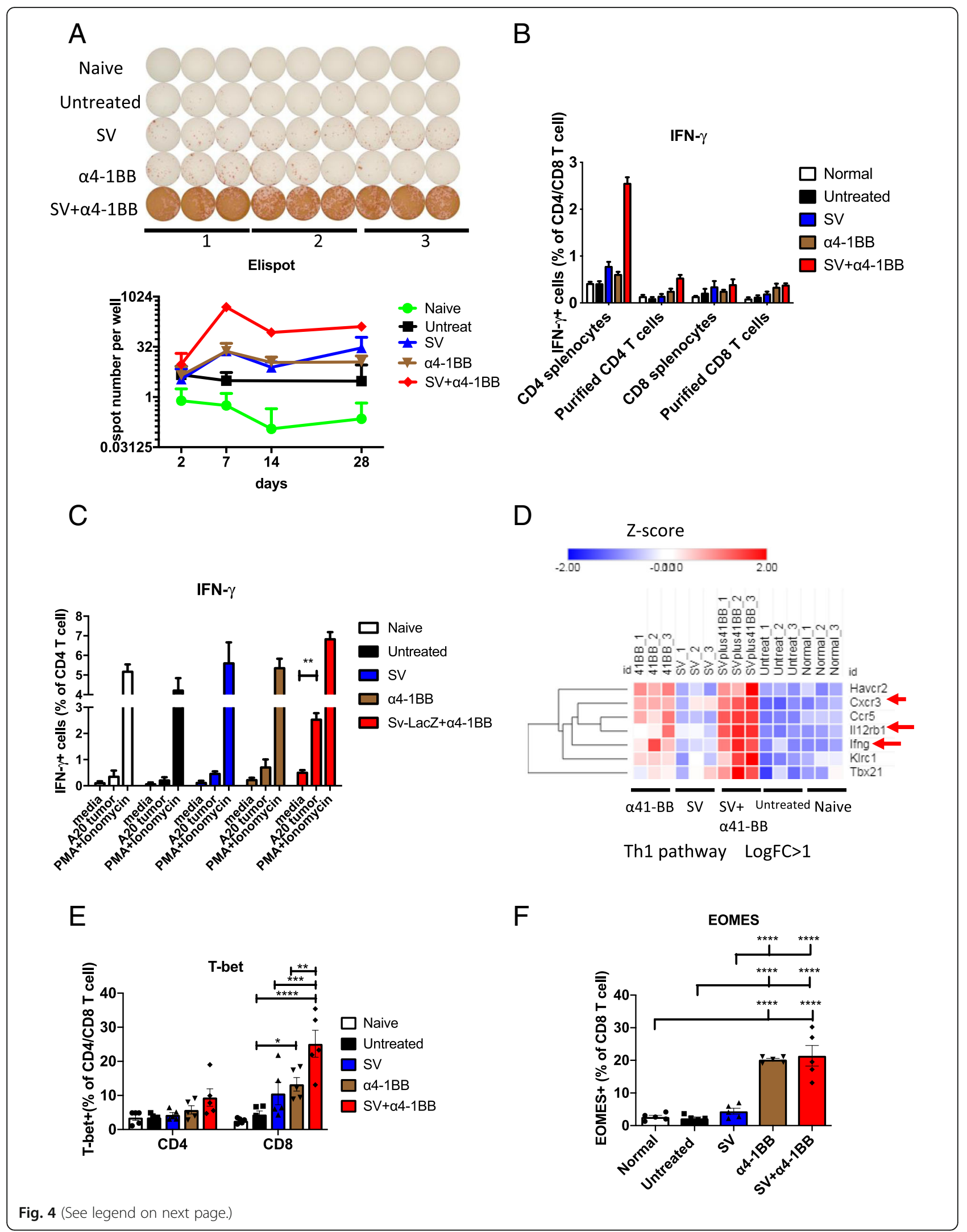


(See figure on previous page.)

Fig. 4 Sindbis virus plus a4-1BB combination induced Th1 differentiation and IFNy production. a IFNy Elispot analysis of splenocytes harvested at day 2, 7, 14 and 28 from all groups as indicated. Upper panel, IFNy Elispot image on day 7 after treatment. 1,2,3: three individual mice. Lower panel, IFNy spots number from indicated groups over the course of treatment $\left(2 \times 10^{5}\right.$ splenocytes per well). No stimulator was added. b IFNy production from CD4/CD8 T cell population in splenocytes and purified CD4/CD8 T cells. All groups were cultured in media for $5 \mathrm{~h}$ in the presence of brefeldin A. c IFNy production from purified CD4 T cells at different stimulation conditions. d Upregulated Th1 pathway gene set under SV, a4-1BB and SV + a4-1BB stimulation. Expression values are shown by Z-score. Genes are hierarchically clustered by one minus Pearson correlation (day 7). e T-bet expression for all groups as indicated. $\mathbf{f}$, EOMES expression for all groups as indicated. CD8 T cell gated. e, f day 7 after treatment. ${ }^{*}, p<0.05 ;{ }^{* *}, p<0.01,{ }^{* * * *}, p<0.0001$

explored whether low doses of $\alpha 4-1 \mathrm{BB} \mathrm{mAb}$ and fewer injections would be as effective in curing tumor bearing mice as the higher doses and frequencies used in our initial stduies. As demonstrated (Additional file 1: Figure S8A and B), A20 tumor bearing mice can be completely cured by SV (3 times per week for 3 weeks) plus a low dose of $\alpha 4-1 \mathrm{BB} \mathrm{mAb}$ $(50 \mu \mathrm{g}$ per week for 3 weeks). This reduces both the $\mathrm{SV}$ and $\alpha 4-1 \mathrm{BB} \mathrm{mAb}$ dosing requirements. The reduced dose of $\alpha 4-1 \mathrm{BB} \mathrm{mAb}$ would be helpful, as well, in preventing the $\alpha 4-1 \mathrm{BB} \mathrm{mAb}$ induced liver toxicity reported by some investigators [36].

\section{All tumor cured mice acquired long lasting antitumor immunity}

To investigate the memory response to A20 lymphoma, naïve and tumor cured mice were inoculated with $3 \times 10^{6}$ A20 tumor cells. Only mice that had survived more than 4 months after 1 st time of tumor challenge were chosen. In all tumor cured mice, we found that A20 lymphoma was completely rejected whereas naïve mice were susceptible to A20 inoculation (Fig. 7a).

To confirm anti-tumor specificity has been elicited, we measured IFN $\gamma$ production of purified $\mathrm{T}$ cells in the presence or absence of tumor cells by Elispot assay. $\mathrm{T}$ cells were isolated from naïve and cured mice under SV plus $\alpha 4-1 B B$ treatment (4 months after treatment finished). Isolated T cells were co-cultured with A20 and CT26 tumor cells respectively. Co-culturing with A20 cells dramatically enhanced IFNy production, whereas co-culturing with CT26 cells only slightly enhanced IFN $\gamma$ production (Fig. 7b).

Next, we measured cytotoxicity to both naïve and cured mice under SV plus $\alpha 4-1 \mathrm{BB}$ treatment (the same method as Fig. 3a). Compared with naïve, cured mice had enhanced cytotoxicity to A20 lymphoma cells, but not to CT26 tumor cells. To confirm that this is mediated by $\mathrm{T}$ cells, the same experiment was done using purified $\mathrm{T}$ cells. Cured mice had enhanced cytotoxicity compared with naïve mice (Fig. 7c).
To better understand differences between this memory $\mathrm{T}$ cell response and the initial treatment responses as observed on day 7, RNA-Seq was performed by using purified splenic $\mathrm{T}$ cells from all rechallanged groups. In $\mathrm{T}$ cells of these rechallened mice we found only a few differentially expressed genes among the three treated groups (Additional file 6: Table S5), indicating that tumor cured mice develop a very similar $\mathrm{T}$ cell gene expression profile regardless of treatment method. Compared with untreated, KEGG analysis indicates that TCR signaling is the highest upregulated pathway in SV plus $\alpha 4-1 \mathrm{BB}$ group (Fig. 7d), indicating that continuously enhanced TCR signaling is critical for maintaining antitumor immunity.

\section{Discussion}

The conventional view of oncolytic virus therapy against tumors is that it requires selective infection of cancer cells resulting in the induction of cancer cell lysis and apoptosis. TAAs, released from dead tumor cells, attract and further stimulate an antitumor immune response. Although A20 lymphoma cells are resistant to infection by $\mathrm{SV}$, these vectors offer an unique opportunity to treat unsusceptiable liquid tumors efficiently. This inducing long-lasting memory/ anti-immunity regardless of infectivity. In previous studies, we demonstrated vectors encoding TAAs, such as NYESO1, could cure CT26-NYESO1 tumors $[6,18]$. Here, we found that encoding a TAA is not necessary for SV vectors plus $\alpha 4-1 \mathrm{BB}$ mAb therapy to be fully successful. SV vectors lacking an A20 lymphoma TAA were able to treat A20 lymphoma and, in combination with $\alpha 4-1 \mathrm{BB} \mathrm{mAb}$, eradicated the growing tumors.

Compared with other cancer immunotherapies, SV therapy has several prominent advantages. Unlike conventional CAR-T, TCR-T or neoantigen specific patient derived $\mathrm{T}$ cells, SV therapy does not require long processing time including cell harvesting, expansion, gene editing and reinfusion. SV therapy also eliminates concerns of graft versus host disease involved in using allogenic T cells. 


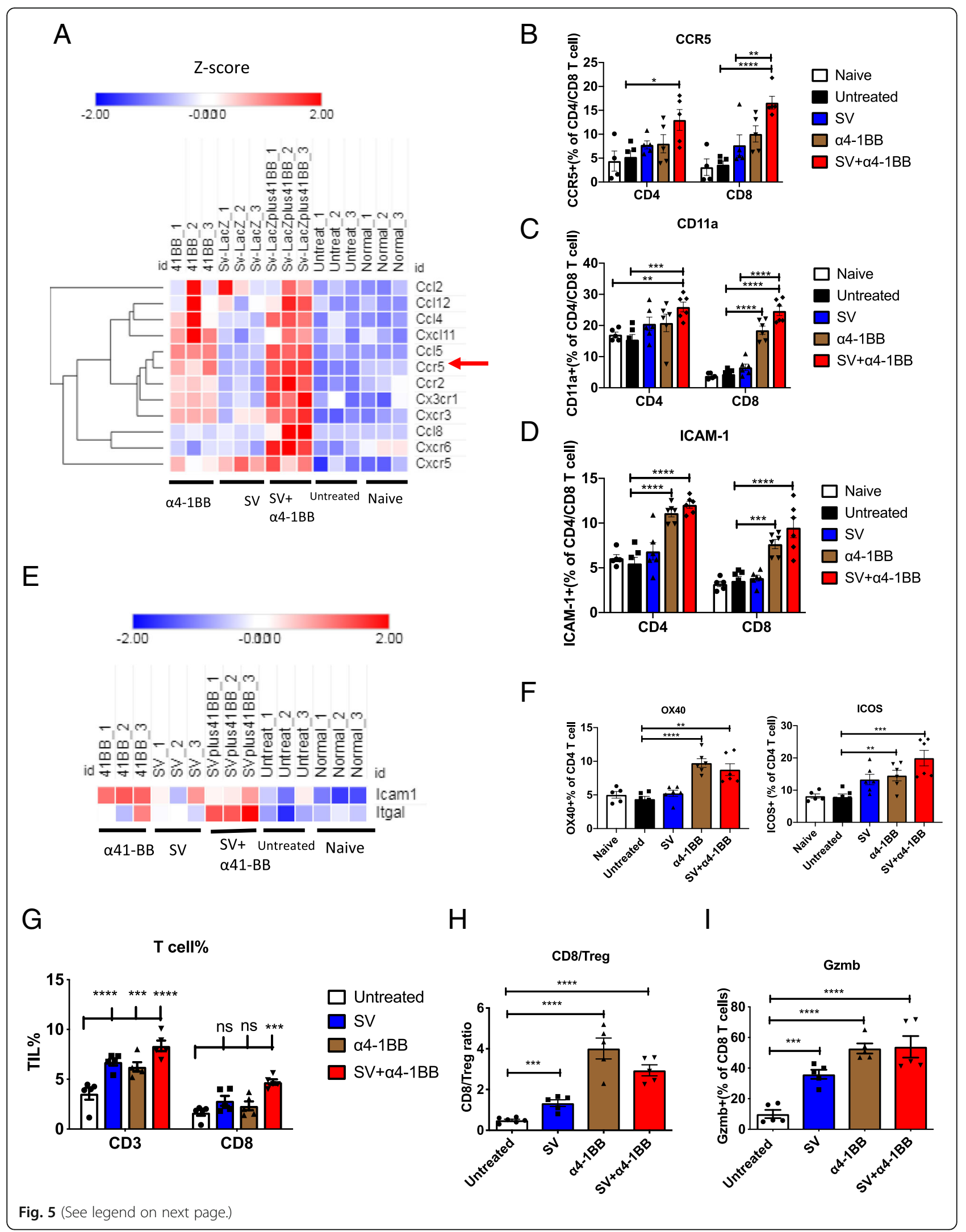


(See figure on previous page.)

Fig. 5 SV and a4-1BB mAb stimulated chemotaxis, adhesion and enhanced T cell infirtration and activation in tumor. a Heat map of the expression pattern of SV + a4-1BB upregulated chemokine and chemokine receptor genes (Expression values are shown by Z-score.) Genes are hierarchically clustered by one minus Pearson correlation (day 7). $\mathbf{b}$ the percentage of CCR5 ${ }^{+}$cells was measured by flow cytometry (day 7). $\mathbf{c}$, $\mathbf{d}$ The percentage of $\mathrm{CD}_{11 \mathrm{a}^{+}}$(c) and ICAM-1+ cells (d) was measured by flow cytometry. e the relative expression of CD11a (Itgal) and ICAM-1 was shown by heat map measured by RNA-Seq. Expression values are shown by Z score. $\mathbf{f}$ The percentage of OX40 ${ }^{+}$and ICOS ${ }^{+} \mathrm{T}_{\text {cells }}$ were measured by flow cytometry. ${ }^{*}, p<0.05 ;^{* *}, p<0.01 ;{ }^{* * *}, p<0.001 ;{ }^{* * * *}, p<0.0001$. g The frequency of CD3 and CD8 T cells to total harvested cells from tumor was measured by flow cytometry. $\mathbf{h}$ CD8/Treg ratio of tumor infirtrated T cells. $\mathbf{i}$ The percentage of granzyme B+ CD $8 \mathrm{~T}$ cells as indicated. *,$p<0.05$; $^{* *}, p<0.01$; $^{* *}, p<0.001$; $^{* * *}, p<0.0001$

SV therapy does not require the incorporation of specific TAAs as does tumor antigen vaccine. This is particularly important when effective immune reactive TAAs are unknown. It is possible that the immunotherapeutic response of SV vectors plus $\alpha 4-1 \mathrm{BB} \mathrm{mAb}$ is independent of whether a tumor is "cold" (i.e., having few TAAs or mutation-specific neoantigens capable of promoting robust $\mathrm{T}$ cell activation) or "hot."

Clinical trials of 2 4-1BB agonist antibodies, urelumab and utomilumab, are underway. Despite initial signs of efficacy, clinical development of urelumab has been hampered by inflammatory liver toxicity at doses $>1 \mathrm{mg} / \mathrm{kg}$ [37]. Utomilumab has a superior safety profile, but is a less potent $4-1 \mathrm{BB}$ agonist relative to urelumab [37]. Both antibodies have demonstrated promising results in patients with lymphoma and are being tested in combination therapy trials with other immunomodulatory agents [37]. The combination of $\alpha 4-1 \mathrm{BB} \mathrm{mAb}$ with other immunomodulatory reagents like $\mathrm{SV}$ vectors might help overcome these limitations and should be explored.

The quick inhibition of tumor growth is critical for cancer therapy because tumor cells undergo exponentially rapid division. However, the induction of adaptive immunity and establishment of tumor specific immunity takes a long time. An ideal therapy requires an early, quick reduction of tumor burden, and a later induction of anti-tumor specificity that prevents relapse. In this study, we proposed that SV plus $\alpha 4$ $1 \mathrm{BB}$ mAb treatment induced massive $\mathrm{T}$ cell activation due to viral induced immune response. This massive activation helps to control the tumor in a TAA nonspecific manner. A similar mechanism has been demonstrated in several other studies. Morphy et al. showed that combining agonistic anti-CD40 with IL-2 induces expansion of highly cytolytic, antigenindependent "bystander-activation" that was responsible for anti-tumor effects [38, 39].

In another study, cytokine was shown to directly induce memory CD8 $\mathrm{T}$ cells expressing NKG2D and granzyme $\mathrm{B}$ and that these $\mathrm{T}$ cells acquire broadly lytic capabilities without cognate antigen engagement [40]. In our system, we found that both NKG2D
(KLRK1) and granzyme B are highly expressed under combination treatment. This massive nonspecific activation is critical for controlling tumor growth at an early time point (day 7). This step is also important for inducing anti-tumor specificity that is mediated by TAAs released from dead tumor cells due to nonspecific killing. After tumor regression, $\mathrm{T}$ cells from treated animals were able maintain the ability to produce IFN $\gamma$ and acquired immunological memory to rapidly reject A20 lymphoma rechallenges. IFN $\gamma$ production from purified $\mathrm{T}$ cells of cured mice was significantly enhanced after encountering A20 tumor cells. This demonstrates that anti-tumor specificity is fully established in cured mice.

Oncolytic vaccinia virus and adenovirus have been used in combination with 4-1BB agonist (either $\alpha 4$ $1 \mathrm{BB} \mathrm{mAb}$ or $\mathrm{OV}$ expressing 4-1BBL) by other investigators. John et al. [41] demonstrated that vaccinia virus and anti-4-1BB combination therapy elicits strong antitumor immunity. However, this combination therapy did not cure mice. While, vaccina virus can infect tumor cells and induce lytic cell death, in our model, SV infection of tumor cells is not necessary. In another vaccinia virus therapy, oncolytic vaccinia virus expressing 4-1BBL has been used for treatment [42]. However the effects were only observed when combined with host lymphodepletion [42]. While lymphodepletion is commonly used in some immunotherapies, it can lead to toxicity, and increased risks of infection. Adenovirus has also been used with $\alpha$ 4-1BB agonist in a combination therapy. Huang et al. [43] showed that tumor specific immunity was induced by the combination of dendritic cells (DC) and oncolytic adenovirus expressing IL-12 and 4-1BBL. DC co-transfer is required for this therapy. The extra cost and time consumption involved in self-DC harvesting, culture and maturation might pose barriers to application of this approach in cancer patients, whereas, our combination therapy does not require any ex-vivo steps. In another study, oncolytic adenovirus armed with CD40L and 4-1BBL were used to treat pancreatic cancer. Again, OV infection of tumor cells and tumor associated stromal cells was a prerequisite 

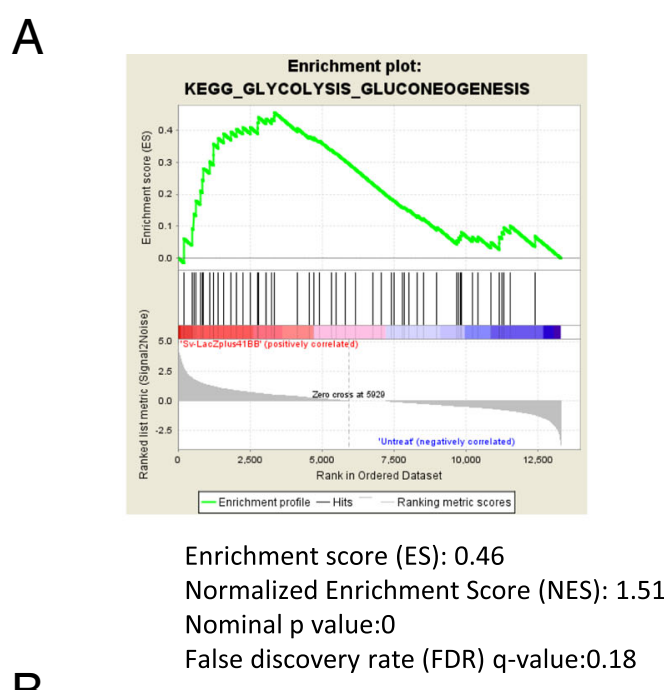

B
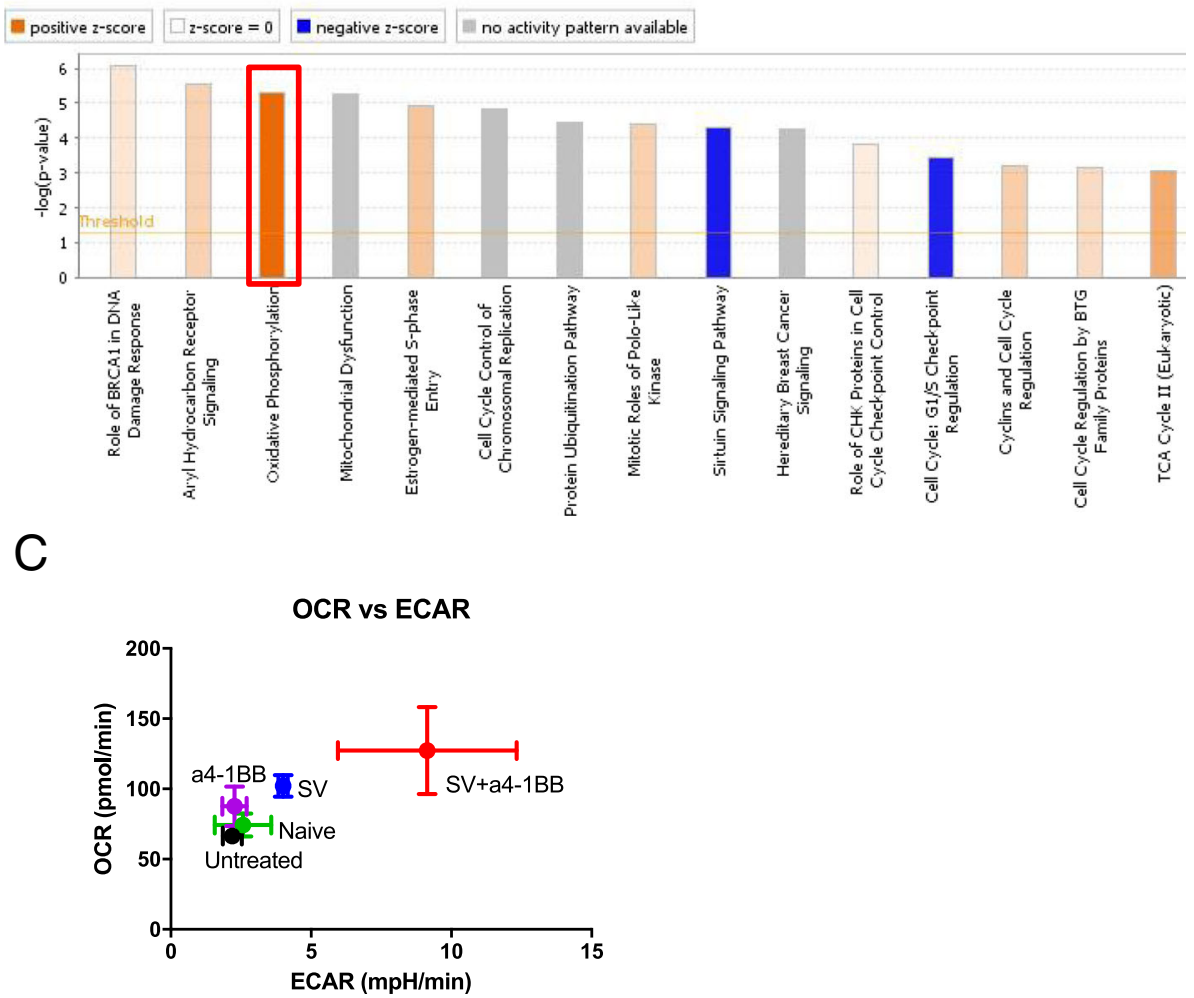

Fig. 6 Sindbis virus plus a4-1BB synergistically enhanced T cell glycolysis and oxidative phosphorylation. a GSEA enrichment plot of KEGG (SV + a4-1BB vs. untreated) glycolysis pathway. $\mathbf{b}$ The canonical pathways of SV plus a4-1BB Ab stimulation are clustered by IPA. Red square, oxidative phosphorylation. c Both oxygen consumption rate (oxidative phosphorylation) and Extracellular Acidification Rate (glycolysis) were measured by seahorse XFe24. All groups are as indicated $(n=4)$

for antitumor effect and immune activation [44], a condition that is not required by SV vectors.

Our study identified and compared the upregulated molecular pathways of responsive $\mathrm{T}$ cells induced by $\mathrm{SV}$ vectors and $\alpha 4-1 \mathrm{BB} \mathrm{mAbs}$ alone and in combination. These observations provide novel insights to guide future studies.
In summary, OV therapy has become a promising therapy for treating cancer. The combination of oncolytic virus and checkpoint inhibitor generally achieve a better therapeutic effect than either alone [18, 45]. $\alpha 4-1 \mathrm{BB}$ mAb has also been used in combination with other therapeutic agents to enhance its antitumor effect $[46,47]$. In this study, we demonstrated that the 
A
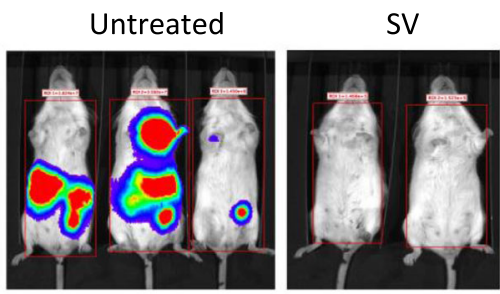

$\alpha 4-1 B B \quad S V+\alpha 4-1 B B$
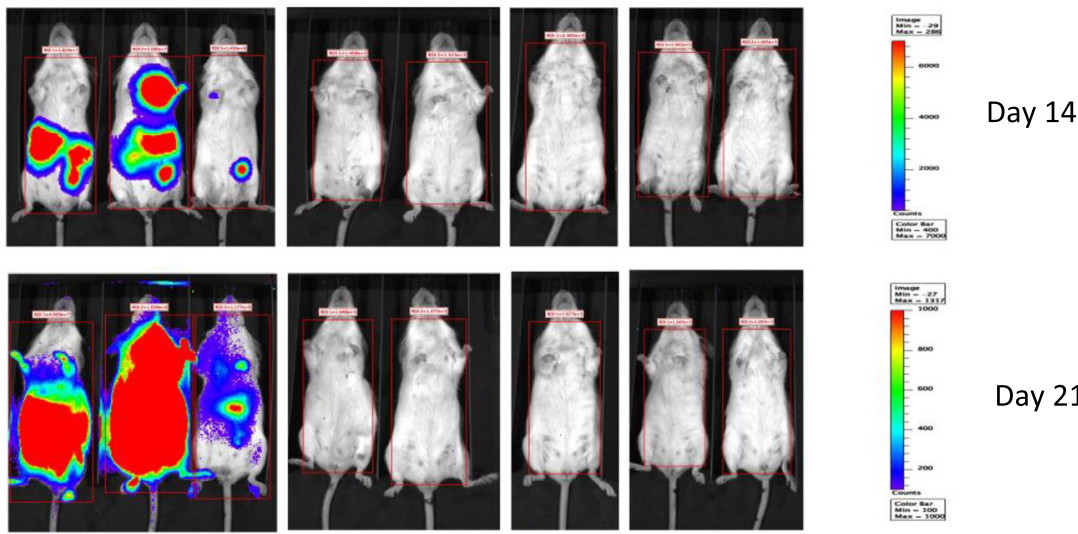

B

C
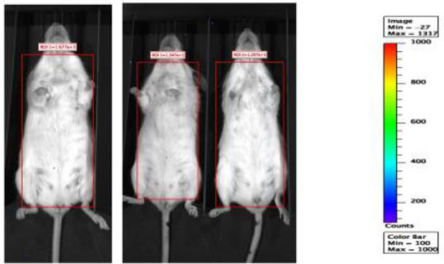

Day 21

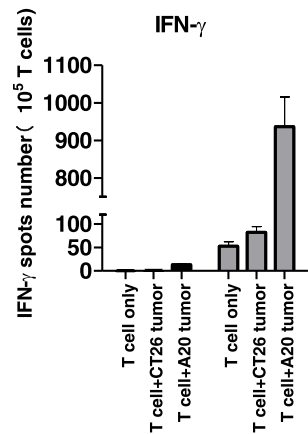

A20 Fluc

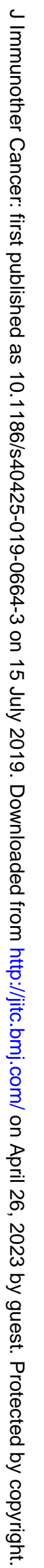

Fig. 7 (See legend on next page.)
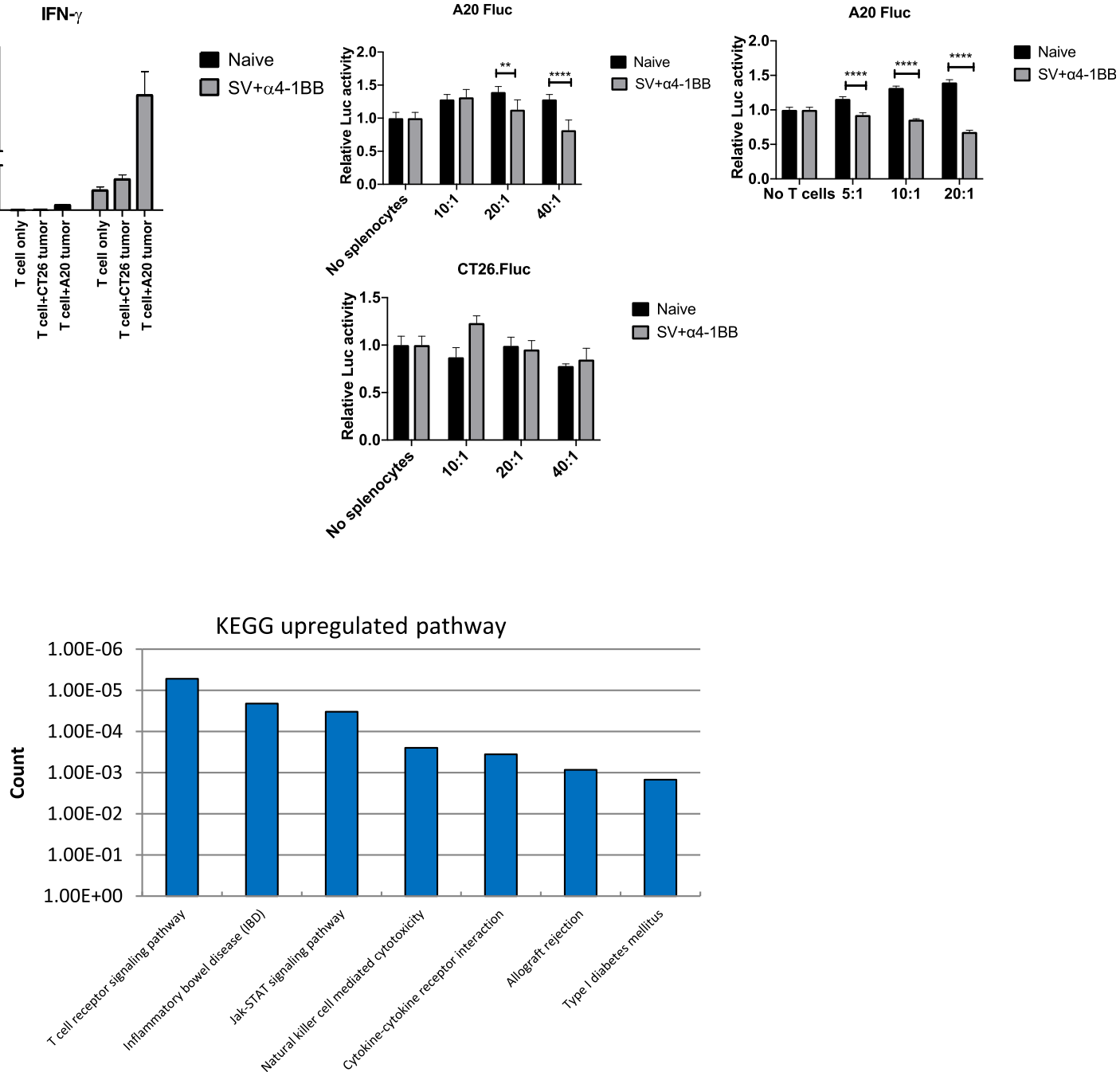

D 
(See figure on previous page.)

Fig. 7 Cured mice are completely protected from A20 lymphoma rechallenge. a, Bioluminescence images of groups, previously treated as indicated, were rechallenged with A20 lymphoma cells. b IFNY production from purified T cells of all groups(To SV + a4-1BB, 4 months after treatment finished), in the absence or presence of A20 tumor cells $\left(5 \times 10^{4}\right.$ per well), was measured by Elispot assay. c Cytotoxicity assay was performed the same as Fig. 3a. Left 2 panels, total splenocytes were used. Right, purified T cells were used. Left upper, A20 Fluc cells and left lower, CT26 Fluc cells were used for co-culture. $\mathbf{d}$, Significant differential (SD) upregulated gene sets are clustered by DAVID KEGG analysis. ${ }^{*}, p<$ $0.05 ; * *, p<0.01 ;{ }^{* * *}, p<0.0001$

combination of SV and $\alpha 4-1 \mathrm{BB}$ mAb has a synergistic effect and represents a potent and robust therapeutic treatment able to cure B lymphomas and provide long term protection in a preclinical model.

\section{Conclusions}

In conclusion, SV vectors in combination with $\alpha 4$ $1 \mathrm{BB} \mathrm{mAb}$ completely eradicated a B-cell lymphoma in a preclinical mouse model, a result that could not be achieved with either treatment alone. Tumor elimination involves a synergistic effect of the combination that significantly boosts $\mathrm{T}$ cell cytotoxicity, IFN- $\gamma$ production, migration, tumor infiltration and oxidative phosphorylation. In addition, all mice that survived after treatment developed long lasting antitumor immunity. Our study provides a novel, alternative method for B cell lymphoma treatment and describes a rationale to help translate SV vectors plus agonistic mAbs into clinical applications.

\section{Additional files}

Additional file 1: Figure S1. A20 lymphoma cells were SV infection resistant. A, A2O cells and BHK cells were infected with SV carrying GFP overnight. GFP expression was observed under fluorescent microscope. B, SV-GFP infectivity to BHK cells was verified by flow cytometry. C, SV-GFP infectivity to A20 cells in vivo were measured by flow cytometry. $10^{7} \mathrm{~A} 20$ cells (express CD45.2) were inoculated to CByJ.SJL(B6)-Ptprca/J (CD45.1 BALB/C) mice. Recipient mice were treated with SV-GFP 4 days later. GFP expression was measured the next day. Figure S2. SV infection enhanced cell cycle progression and migration. A, DAVID KEGG analysis. B, GSEA enrichment plot of KEGG (SV vs. Untreated) cell cycle pathway (SV vs. Untreated). C, cell movement pathway was signficiantly enhanced by IPA(SV vs. Untreated). Figure S3. Significant differential (SD) upregulated genes are clustered by DAVID analysis. Figure $\mathbf{S 4}$. Untreated group had low ratio of $T$ cells and high ratio of regulatory $T$ cells on day 28 . The frequency of CD4 (A), CD8 (B), Treg (C) were measured by flow cytometry. D, Treg/ CD8 ratio as indicated. Figure S5. IFNY production from splenocytes of all groups with or without tumor inoculation on day 7 after treatment was measured by Elispot. With tumor: tumor was inoculated on day 0 . Without tumor: tumor was not inoculated. No stimulator was added in Elispot assay. Figure S6. IFNy production measurement. A, IFNY production (at day 7) by all groups, as indicated, was measured by Elispot. B, IFNy production of purified T cells (CD8 T cell portion) on day 7 after treatment was measured by flow cytometry. Figure S7. The phenotype of tumor infirtrated T cells. A-E, The percentage of Ki67+, Foxp3+, T-bet+, EOMES+, NKG2D+ T cells were measured by flow cytometry. Figure $\mathbf{8 8}$. SV plus low dose a4-1BB mAb cured A20 tumor bearing mice. (PPTX $9838 \mathrm{~kb})$

Additional file 2: Table S1. The SD expressed genes list for SV vs. untreated group by RNA-Seq $(q<0.05, \log 2 F C \geq 1$ and $\log 2 F C \leq-1)$. (XLSX $24 \mathrm{~kb})$
Additional file 3: Table S2. The upregulated cell movement pathway for SV vs. untreated group by IPA. SV induced SD upregulated gene sets are clustered by DAVID analysis (SV vs. Untreated). Gene clusters are ranked by enrichment score. (XLSX $9 \mathrm{~kb}$ )

Additional file 4: Table S3. The SD expressed genes list for SV + a4$1 \mathrm{BB}$ vs. untreated group by RNA-Seq $(\mathrm{q}<0.05, \log 2 \mathrm{FC} \geq 1$ and $\log 2 \mathrm{FC} \leq$ - 1). (XLSX $113 \mathrm{~kb})$

Additional file 5: Table S4. The SD expressed genes list for SV + a4$1 \mathrm{BB}$ vs. SV group by RNA-Seq $(\mathrm{q}<0.05, \log 2 \mathrm{FC} \geq 1$ and $\log 2 \mathrm{FC} \leq-1)$. (XLSX $52 \mathrm{~kb}$ )

Additional file 6: Table S5. The SD expressed gene lists among all tumor cured mice groups. (XLSX $10 \mathrm{~kb})$

\section{Abbreviations}

APC: Antigen presentation cell; DAVID: The Database for Annotation, Visualization and Integrated Discovery; ECAR: Extracellular acidification rate; GSEA: Gene Set Enrichment Analysis; IPA: Ingenuity pathway analysis; KEGG: Kyoto Encyclopedia of Genes and Genomes; OCR: Oxygen consumption rate; OV: Oncolytic virus; SD: Significant differential; SV: Sindbis virus; TAA: Tumor associated antigen; TIL: Tumor infiltrating lymphocyte

\section{Acknowledgements}

The authors are grateful to Dr. Christine Pampeno for careful review of the manuscript. The authors also thanks for Dr.Alicia Hurtado Martinez, Ms. Feng Shanshan for technical support.

\section{Authors' contributions}

MY and DM contributed to the experimental design and conception. MY acquired the data. MY, IS and SO did the experiments. MY analyzed and summarized data. MY and DM wrote the manuscript. MY, IS, AT and DM reviewed and revised the manuscript. All authors read and approved the final manuscript.

\section{Funding}

Funding was provided by NIH grant 5R44CA206606 and through a Research and Licensing Agreement between NYU Langone and Cynvec, which licenses the Sindbis technology to Cynvec.

\section{Availability of data and materials}

The datasets used and/or analyzed during the current study are available from the corresponding author on reasonable request.

\section{Ethics approval and consent to participate}

All animal studies were conducted with prior approval from the New York University Medical Center Animal Care and Use Committee and in a facility accredited by the American Association for Accreditation of Laboratory Animal Care.

\section{Consent for publication}

All authors read and approved the final manuscript.

\section{Competing interests}

The authors declare that they have no competing interests. 
Received: 27 February 2019 Accepted: 5 July 2019

Published online: 15 July 2019

\section{References}

1. de Winde CM, Elfrink S, van Spriel AB. Novel insights into membrane targeting of B cell lymphoma. Trends Cancer. 2017;3:442-53.

2. Anwer $F$, Shaukat $A A$, Zahid $U$, et al. Donor origin CAR T cells: graft versus malignancy effect without GVHD, a systematic review. Immunotherapy. 2017;9:123-30

3. Graham C, Jozwik A, Pepper A, Benjamin R. Allogeneic CAR-T Cells: More than Ease of Access? Cells. 2018;7(10):155.

4. Lundstrom K. Oncolytic Alphaviruses in Cancer Immunotherapy. Vaccines (Basel). 2017:5(2):9.

5. Marelli G, Howells A, Lemoine NR, Wang Y. Oncolytic viral therapy and the immune system: a double-edged sword against Cancer. Front Immunol. 2018;9:866.

6. Granot T, Yamanashi Y, Meruelo D. Sindbis viral vectors transiently deliver tumor-associated antigens to lymph nodes and elicit diversified antitumor CD8+ T-cell immunity. Mol Ther. 2014;22:112-22.

7. Raja J, Ludwig JM, Gettinger SN, Schalper KA, Kim HS. Oncolytic virus immunotherapy: future prospects for oncology. J Immunother Cancer. 2018; 6:140

8. Tseng JC, Levin B, Hurtado A, et al. Systemic tumor targeting and killing by Sindbis viral vectors. Nat Biotechnol. 2004;22:70-7.

9. Lloyd G. Alphaviruses. In: Principles and practices of clinical virology. 6th ed. Chichester: Wiley; 2009

10. Brummer-Korvenkontio M, Vapalahti O, Kuusisto $P$, et al. Epidemiology of Sindbis virus infections in Finland 1981-96: possible factors explaining a peculiar disease pattern. Epidemiol Infect. 2002;129:335-45.

11. Manni T, Kurkela S, Vaheri A, Vapalahti O. Diagnostics of Pogosta disease: antigenic properties and evaluation of Sindbis virus IgM and IgG enzyme immunoassays. Vector Borne Zoonotic Dis. 2008;8:303-11.

12. Strauss JH, Strauss EG. The alphaviruses: gene expression, replication, and evolution. Microbiol Rev. 1994;58:491-562.

13. Hardwick JM, Levine B. Sindbis virus vector system for functional analysis of apoptosis regulators. Methods Enzymol. 2000;322:492-508.

14. Hurtado A, Tseng JC, Meruelo D. Gene therapy that safely targets and kills tumor cells throughout the body. Rejuvenation Res. 2006;9:36-44.

15. Strauss JH, Wang KS, Schmaljohn AL, Kuhn RJ, Strauss EG. Host-cell receptors for Sindbis virus. Arch Virol Suppl. 1994;9:473-84.

16. Tseng JC, Zheng Y, Yee H, Levy DE, Meruelo D. Restricted tissue tropism and acquired resistance to Sindbis viral vector expression in the absence of innate and adaptive immunity. Gene Ther. 2007:14:1166-74.

17. Houot R, Goldstein MJ, Kohrt HE, et al. Therapeutic effect of CD137 immunomodulation in lymphoma and its enhancement by Treg depletion. Blood. 2009;114:3431-8.

18. Scherwitzl I, Hurtado A, Pierce CM, Vogt S, Pampeno C, Meruelo D. Systemically administered Sindbis virus in combination with immune checkpoint blockade induces curative anti-tumor immunity. Mol Ther Oncolytics. 2018:9:51-63.

19. Love Ml, Huber W, Anders S. Moderated estimation of fold change and dispersion for RNA-seq data with DESeq2. Genome Biol. 2014;15:550.

20. Huang da W, Sherman BT, Lempicki RA. Bioinformatics enrichment tools: paths toward the comprehensive functional analysis of large gene lists. Nucleic Acids Res 2009;37: 1-13.

21. da Huang W, Sherman BT, Lempicki RA. Systematic and integrative analysis of large gene lists using DAVID bioinformatics resources. Nat Protoc. 2009;4: 44-57.

22. Subramanian A, Tamayo P, Mootha VK, et al. Gene set enrichment analysis: a knowledge-based approach for interpreting genome-wide expression profiles. Proc Natl Acad Sci U S A. 2005:102:15545-50.

23. Leonard WJ, Wan CK. IL-21 Signaling in Immunity. F1000Res. 2016:5:F1000 Faculty Rev-224.

24. Rowell JF, Griffin DE. The inflammatory response to nonfatal Sindbis virus infection of the nervous system is more severe in $S J L$ than in BALB/C mice and is associated with low levels of IL-4 mRNA and high levels of IL-10producing CD4+ T cells. J Immunol. 1999;162:1624-32.

25. Metcalf TU, Baxter VK, Nilaratanakul V, Griffin DE. Recruitment and retention of $B$ cells in the central nervous system in response to alphavirus encephalomyelitis. J Virol. 2013;87:2420-9.
26. Jacobson NG, Szabo SJ, Weber-Nordt RM, et al. Interleukin 12 signaling in T helper type 1 (Th1) cells involves tyrosine phosphorylation of signal transducer and activator of transcription (stat)3 and Stat4. J Exp Med. 1995; 181:1755-62.

27. Pizzoferrato E. B7-2 expression above a threshold elicits anti-tumor immunity as effective as interleukin-12 and prolongs survival in murine Bcell lymphoma. Int J Cancer. 2004;110:61-9.

28. Zhu Y, Ju S, Chen $\mathrm{E}$, et al. T-bet and eomesodermin are required for $\mathrm{T}$ cellmediated antitumor immune responses. J Immunol. 2010;185:3174-83.

29. Gonzalez-Martin A, Mira E, Manes S. CCR5 in cancer immunotherapy: more than an "attractive" receptor for T cells. Oncoimmunology. 2012;1:106-8.

30. Walling BL, Kim M. LFA-1 in T cell migration and differentiation. Front Immunol. 2018:9:952.

31. Sabatos CA, Doh J, Chakravarti S, et al. A synaptic basis for paracrine interleukin-2 signaling during homotypic T cell interaction. Immunity. 2008; 29:238-48.

32. Gerard A, Khan $O$, Beemiller $P$, et al. Secondary $T$ cell- $T$ cell synaptic interactions drive the differentiation of protective CD8+ T cells. Nat Immunol. 2013;14:356-63.

33. Croft M, So T, Duan W, Soroosh P. The significance of OX40 and OX40L to T-cell biology and immune disease. Immunol Rev. 2009;229:173-91.

34. Wahl DR, Byersdorfer CA, Ferrara JL, Opipari AW Jr, Glick GD. Distinct metabolic programs in activated T cells: opportunities for selective immunomodulation. Immunol Rev. 2012;249:104-15.

35. van der Windt GJ, Pearce EL. Metabolic switching and fuel choice during Tcell differentiation and memory development. Immunol Rev. 2012:249:2742.

36. Bartkowiak $T$, Jaiswal $A R$, Ager $C R$, et al. Activation of 4-1BB on liver myeloid cells triggers hepatitis via an Interleukin-27-dependent pathway. Clin Cancer Res. 2018;24:1138-51.

37. Chester C, Sanmamed MF, Wang J, Melero I. Immunotherapy targeting 41BB: mechanistic rationale, clinical results, and future strategies. Blood. 2018 131:49-57.

38. Murphy WJ, Welniak $L$, Back $T$, et al. Synergistic anti-tumor responses after administration of agonistic antibodies to CD40 and IL-2: coordination of dendritic and CD8+ cell responses. J Immunol. 2003;170:2727-33.

39. Monjazeb AM, Tietze JK, Grossenbacher SK, et al. Bystander activation and anti-tumor effects of CD8+ T cells following Interleukin-2 based immunotherapy is independent of CD4+ T cell help. PLoS One. 2014;9: e102709.

40. Tietze JK, Wilkins DE, Sckisel GD, et al. Delineation of antigen-specific and antigen-nonspecific CD8(+) memory T-cell responses after cytokine-based cancer immunotherapy. Blood. 2012;119:3073-83.

41. John LB, Howland L, Flynn JK, et al. Oncolytic virus and anti-4-1BB combination therapy elicits strong antitumor immunity against established cancer. Cancer Res. 2012;72:1651-60.

42. Kim HS, Kim-Schulze S, Kim DW, Kaufman HL. Host lymphodepletion enhances the therapeutic activity of an oncolytic vaccinia virus expressing 4-1BB ligand. Cancer Res. 2009;69:8516-25.

43. Huang JH, Zhang SN, Choi KJ, et al. Therapeutic and tumor-specific immunity induced by combination of dendritic cells and oncolytic adenovirus expressing IL-12 and 4-1BBL. Mol Ther. 2010;18:264-74.

44. Eriksson E, Milenova I, Wenthe J, et al. Shaping the tumor stroma and sparking immune activation by CD40 and 4-1BB signaling induced by an armed oncolytic virus. Clin Cancer Res. 2017:23:5846-57.

45. Quetglas JI, Labiano S, Aznar MA, et al. Virotherapy with a Semliki Forest virus-based vector encoding IL12 synergizes with PD-1/PD-L1 blockade. Cancer Immunol Res. 2015;3:449-54.

46. Souza-Fonseca-Guimaraes F, Blake SJ, Makkouk A, Chester C, Kohrt HE, Smyth MJ. Anti-CD137 enhances anti-CD20 therapy of systemic B-cell lymphoma with altered immune homeostasis but negligible toxicity. Oncoimmunology. 2016;5:e1192740.

47. Hebb JPO, Mosley AR, Vences-Catalan F, et al. Administration of low-dose combination anti-CTLA4, anti-CD137, and anti-OX40 into murine tumor or proximal to the tumor draining lymph node induces systemic tumor regression. Cancer Immunol Immunother. 2018:67:47-60.

\section{Publisher's Note}

Springer Nature remains neutral with regard to jurisdictional claims in published maps and institutional affiliations. 